\title{
SDN-Based Channel Assignment Algorithm for Interference Management in Dense Wi-Fi Networks
}

\author{
M. Seyedebrahimi, F. Bouhafs, A. Raschellà, M. Mackay, Q. Shi \\ Department of Computer Science \\ Liverpool John Moores University \\ Liverpool, United Kingdom \\ e-maill: [M.Seyedebrahimi, F.Bouhafs, A.Raschella, M.I.Mackay, Q.Shi]@ljmu.ac.uk
}

\begin{abstract}
The popularity of Wi-Fi-enabled devices alongside the growing demand for non-licensed spectrum, has made the WiFi networks exceedingly congested. This endangers the efficiency of Wi-Fi and negatively affect the users' experience. The problem is especially pressing in dense areas (e.g. shopping centers) where Wi-Fi channel assignment is more likely to be uncoordinated and the working environment of Wi-Fi Access Points (APs) has become increasingly time-variant. As a result, the availability of SoftwareDefined Networking (SDN) and network virtualization technologies has motivated the use of centralized resource management as a solution. This paper provides an algorithm for channel assignment functionality in the context of SDN-based centralized resource management, which captures the live status of a Wi-Fi network and is capable of optimising the Radio Frequency (RF) channel assignment process. The APs' network arrangement, the current assignment of the channels and the characteristics of the RF channels in IEEE 802.11 have all been taken into account in the proposed model. The performance of the proposed model in terms of the level of the interference, the spectral efficiency at each AP and the Signal to Interference plus Noise Ratio (SINR) at the user-side is evaluated through simulation and compared against state of the art solutions.
\end{abstract}

Keywords-Wi-Fi; channel assignment algorithm; IEEE 802.11; Optimisation; wireless communications

\section{INTRODUCTION}

The last few years have witnessed a massive increase in the popularity of portable devices, such as smartphones and tablets thanks to their functionality, user-friendly interface, and affordable price. This situation has created a growing demand on the wireless spectrum in general, but more specifically $\mathrm{Wi}$ Fi. Due to its utilization of non-licensed frequency bands, Wi-Fi is now facing significant spectrum efficiency issues especially in dense areas such as apartment blocks, and shopping malls where neighboring Wi-Fi Access Points (APs) interfere with each other while competing for the spectrum. This interference between Wi-Fi networks can negatively affect users' experience.

Wi-Fi APs interference is major research challenge that has been studied extensively in the literature with a focus on channel assignment to address the problem. In [1], the authors proposed an approach where an AP can select a suitable channel based on the neighboring APs it detects and its local evaluation of the least congested scanned channels. In [2], the authors proposed a heuristic algorithm that assigns a channel to an AP by analyzing the effect of partially overlapping channels on the Wi-Fi network throughput. In [3], the authors formulated optimisation of channel assignment as a graph colouring problem [4]. However, these solutions do not consider other performance related measurements at the AP such as Signal to Interference plus Noise Ratio (SINR) and interference levels. Although these network-side measurements have been considered in the contributions presented in [5] and [6], the impact of these measurements on the performance of these solutions is limited as they are taken locally and not across all the interference environment.

Although there are a number of centralised $\mathrm{Wi}-\mathrm{Fi}$ management solutions available in the market that provide a global control approach, most of these solutions lack the flexibility to adapt to the dynamic nature of Wi-Fi networks. In addition, these solutions are generally proprietary, which makes it difficult to extend their functionality to address spectrum efficiency. More recently, Software-Defined Networking (SDN) [7] has emerged as an efficient and flexible alternative for data network management including wireless local area networks (WLANs). Frameworks such as OpenSDWN [8], EmPOWER [9], and Odin [10] have been developed according to the SDN concept allowing operators to centralize the network management operations at a single entity, often referred to as a Controller.

Building on this latest development, we propose a channel assignment algorithm as part of our work in the H2020 Wi-5 project [11] that addresses spectrum congestion in Wi-Fi networks using SDN. In this work we propose a novel channel assignment that runs in the SDN controller and that relies on the network monitoring information the controller obtains in order to analyze and calculate the optimised channel assignment configuration across all the Wi-Fi networks.

The rest of the paper is organized as follows: in Section II we present the SDN-based Wi-Fi management framework, and the assumptions made while designing the proposed channel assignment algorithm. In Section III, we formulate the channel assignment optimisation problem and we describe the Channel Assignment algorithm and its implementation. In Section IV we highlight certain considerations that need to be taken into account while implementing and running the algorithm. In Section V we assess our proposed algorithm and present the obtained results, and finally our conclusion is presented in Section VI.

\section{SDN-BASED WI-FI MANAGEMENT FRAMEWORK}

The choice of SDN as a framework to manage dense Wi-Fi environments is justified by the flexibility and cross-layer management approach offered by this concept. Since the 


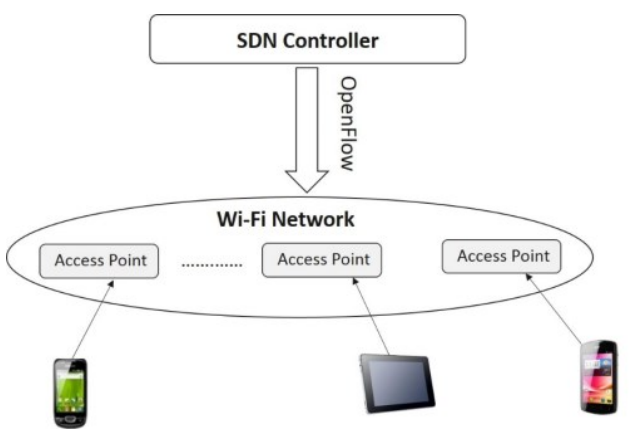

Fig. 1: SDN-Based Wi-Fi Network Management

controller is able to obtain networking monitoring information through the Openflow protocol, it will also be able to decide on the best configuration it needs to apply to the entire network. This has been recently extended to support Wi-Fi related monitoring information in frameworks such as Odin and EmPOWER. In our work, we assume a large Wi-Fi network that consists of $N$ APs, controlled by an SDN Controller, similarly to the Wi-Fi SDN-based management approach proposed in [10], as illustrated in Fig. 1.

Our channel assignment algorithm will be located in the SDN controller and will be triggered every time the controller detects that the interference level has reached a specific threshold. In order to achieve this, the controller relies on periodic network status information it obtains from the network. Once the channel assignment algorithm is executed, the controller applies the channel assignment configuration, as illustrated in Fig. 2.

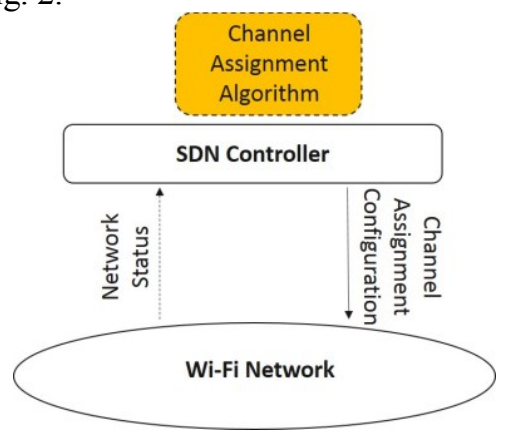

Fig. 2: Channel Assignment using SDN-based Wi-Fi Management Framework

\section{SDN-BASED CHANNEL ASSIGNMENT ALGORITHM}

In our work, we consider $N$ Wi-Fi APs, based on IEEE802.11, that operate on $F$ RF channels. The proposed SDNbased Channel Assignment technique relies on the following information:

1. The topology of the network and the arrangement of the APs.

2. The current channels assignment across all APs

3. The characteristics of the IEEE 802.11 RF channels and their impact on the interference due to a combination of orthogonality and overlapping of the channels.
We define $G^{N \times N}$ as the network topology matrix where:

$$
g_{i j}=\left\{\begin{array}{c}
1, \quad \begin{array}{c}
\text { average power strenght of } A P_{i} \text { around } A P_{j} \\
\text { exceeds a threshold }
\end{array} \\
0, \quad \text { otherwise }
\end{array}\right.
$$

We define $A^{F \times N}$ as the channel assignment matrix where:

$$
a_{i j}=\left\{\begin{array}{lc}
1, & \text { if channel } i \text { is assigned to } A P_{j} \\
0, & \text { otherwise }
\end{array}\right.
$$

We also define $I \in \mathbb{R}^{N \times F}$, the matrix of the interference predicted for $N$ APs and $F$ available channels, where $I_{i, j}$ is the interference level detected within the network as a result of assigning channel $j$ to AP $i$. Finally, we define $U$ as an objective function that represents the interference levels detected by all APs due to their current channel assignment configuration, and that can be formulated as following:

$$
W=G \times A^{T}, \quad U=W . I
$$

Where ' $x$ ' represents the matrix multiplication and ', denotes element-wise multiplication of the matrices. $U$ actually is obtained by taking into account the arrangement of the APs, reflected in $G$, and the channel assignment represented by $A$ alongside the actual impact of the channel selection at each AP from the interference point of view represented by $I$. Since $I$ is a matrix with real values $\left(I \in \mathbb{R}^{N \times \mathrm{F}}\right), U$ in (3) will also be a real matrix $\left(U \in \mathbb{R}^{N \times \mathrm{F}}\right)$. We can describe $U$ as an objective function which represents the magnitude of the interference in the whole system and encompasses the AP inter-relations, through $G$, and the scale by which APs are conflicting with each other in each channel represented by $G \times A^{T}$ as follows:
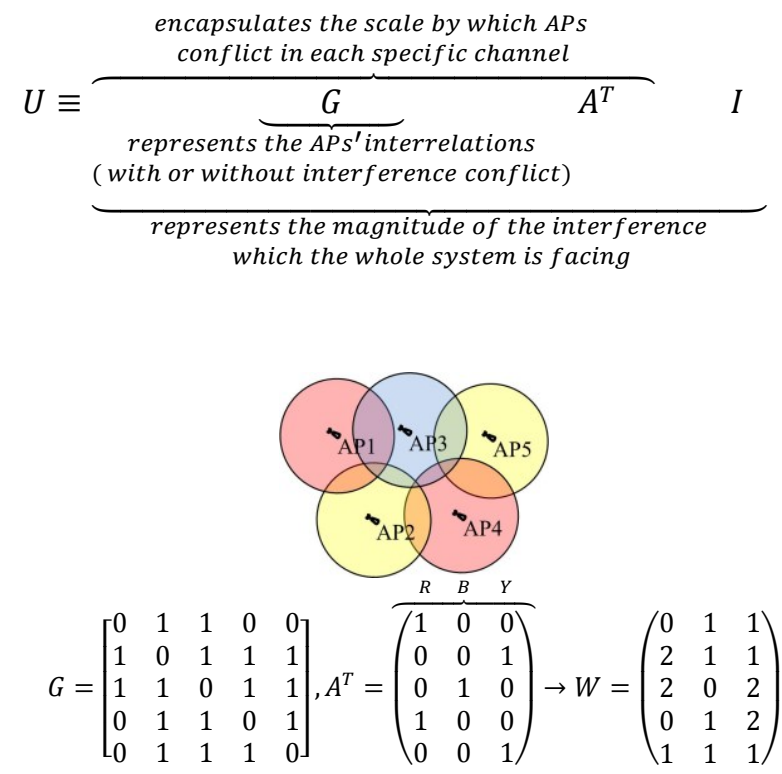

Fig. 3 An example describing the application of G, A and W 
To clarify this concept, a simple network with $N=5$ APs and $F=3$ RF channels (denoted as Red, Blue and Yellow colours) is shown in Fig. 3. We assume that AP1 is not in the contention range of AP4 and AP5, hence the corresponding elements in $G$ will be zero. Matrix $A$ represents the allocated channels to the APs and $W=G \times A^{T}$ embodies the scale of the conflict at each AP with regards to each channel. For example, given the channel assignment and the arrangement of AP1, AP2, AP3 and AP5, $W_{41}=0$ indicates no conflict for the channel denoted as Red to be assigned to AP4 while the value $W_{43}=2$ indicates a higher scale of the conflict if the channel denoted as Yellow be assigned to AP4. By applying the actual interference of the channels considering their overlap and orthogonality through $I$ in (3), the objective function $U$ provides the network-wide interference quantities which need to be minimized through an optimised channel assignment.

We define $A^{*}$ as the optimised channel assignment matrix that provides the minimum interference levels throughout the network and can be obtained as follows:

$$
A^{*}=\min _{A} \sum_{i \leq N} \sum_{j \leq \mathrm{F}} u_{i, j}
$$

power and interference values, therefore the elements of $U$ in (5), are considered to be real values not converted into $\mathrm{dB}$. The channel assignment optimisation problem can be expressed by:

$$
\left\{\begin{array}{c}
A^{*}=\min _{A} \sum_{\text {all }} G \times A^{T} . I \\
A \in\{0,1\}^{F \times N} \\
|| A(:, i) \|\left.\right|_{1}=1 \\
\forall a_{i j} \in A: \sum \sum a_{i j}=N
\end{array}\right.
$$

Where $\|.\|_{1}$ represents 1 -norm, the summation of the elements in each column of $A$. The constraints in (6) are based on the fact that each AP will be assigned only one channel and in total exactly $N$ channel selections should be made for $N$ APs.

$$
A=\left(\begin{array}{ccc}
a_{11} & \cdots & a_{1 N} \\
\vdots & \ddots & \vdots \\
a_{F 1} & \cdots & a_{F N}
\end{array}\right) \rightarrow x=\left(\begin{array}{c}
x_{1} \\
\vdots \\
x_{N} \\
\vdots \\
x_{(F-1) \times N+1} \\
\vdots \\
x_{F \times N}
\end{array}\right) \equiv\left(\begin{array}{c}
a_{11} \\
\vdots \\
a_{1 N} \\
\vdots \\
a_{F 1} \\
\vdots \\
a_{F N}
\end{array}\right)
$$

By element-wise multiplication of matrices in (6) and representing all elements of matrix $A$ in the form of a vector of unknown values, $x \in\{0,1\}^{N * F \times 1}$, as shown in equation (7), the optimisation problem presented in (6) can be solved using a binary Integer Linear Programing (ILP) as follows:

$$
\left\{\begin{array}{c}
x^{*}=\min _{x} c^{T} x \\
B x=b \\
x_{i} \in[0,1], \quad \forall i: 1 \leq i \leq N \times F
\end{array}\right.
$$

$$
\begin{aligned}
& c=f(G, I), \quad b=1^{N \times 1},
\end{aligned}
$$

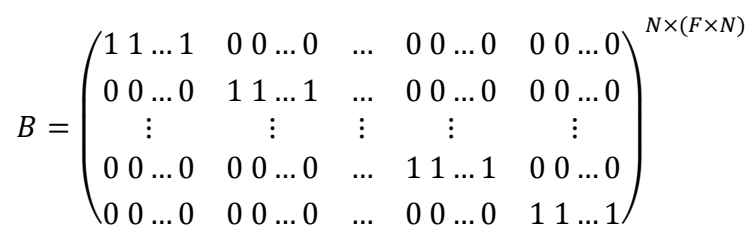

Where $c$ is a coefficient matrix resulting from the element-wise expansion of (6), and $x$ provides the desired channel assignment (i.e. the elements of matrix $A^{*}$ ). The channel assignment matrix $A$, in (6), has just one non-zero value in each row corresponding to the assigned channel. Matrix $B$ reflects the constraints defined for $A$ in (6) over the values of $x$ in (8).

\section{IMPLEMENTATION CONSIDERATIONS USING THE SDN- BASED WI-FI MANAGEMENT FRAMEWORK}

In order to find the best channel assignment configuration that will result in minimal interference within a dense Wi-Fi environment, the ILP algorithm described in (8) can be run only once and needs to include all APs, as depicted in Fig. 4(a). The central location of the controller allows it to obtain a global view of the network status, through the regular updates it obtains from all APs. However, since the ILP algorithm will run only once, the APs will not have the chance to measure the interference levels for each channel assignment configuration. In this case, the value of the interference matrix $I$ needs to be predicted using the initial network measurements collected by the SDN controller. Once an optimal channel assignment configuration is found, the SDN controller applies this configuration through its Openflow southbound API. As mentioned previously, the algorithm is executed every time the interference level reaches a certain threshold. This is particularly useful when considering the dynamic nature of Wi-Fi networks, where the topology of the network or the transmission powers of some APs might change.

In most cases, including the initial setup of the network or during the re-initialization of the network, the required information to create the whole matrices $G$ and $I$ cannot be fully achieved or predicted adequately. Therefore, the practical approach will be to expand the analytical model, upon which the channel assignment algorithm is based, gradually, as depicted in Fig. 4(b). Hence, the channel assignment procedure starts by assigning a channel (e.g. one of the orthogonal channels 1, 6 or 11) to one of the APs, and then continue adding other APs to the graph matrix one by one and solving (8) every time the matrix is updated. The previously assigned APs in each run update the latest relevant information needed for $I$ in the next run, hence a reasonable timing gap between two runs of the algorithm is needed. The proposed algorithm also provides the possibility of assigning/re-assigning the channels for a single AP (Fig. 4(c)) e.g., in the case of channel assignment to a newly activated AP. It also can be employed for assigning just a subset of the APs while some other APs are excluded from the assignment process (Fig. 4(d)). This is either due to inaccessibility of the APs at the controller, or for reducing a possible disruptive effect in the network due to recurrent channel assignments whenever necessary. 


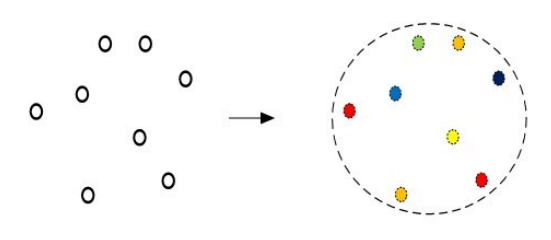

(a) Optimization in one run given the graph for the whole network

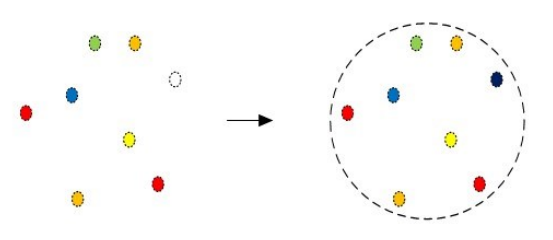

(c) Running optimization process just for a single AP

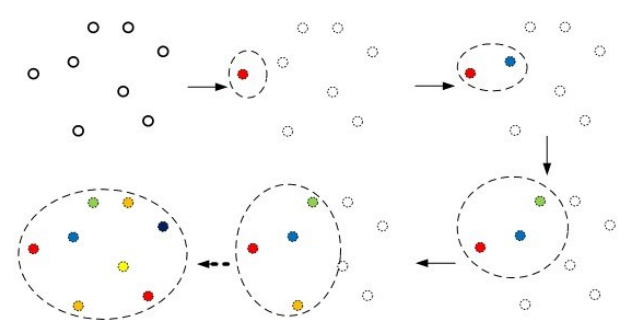

(b) Optimization of a gradually expanding network

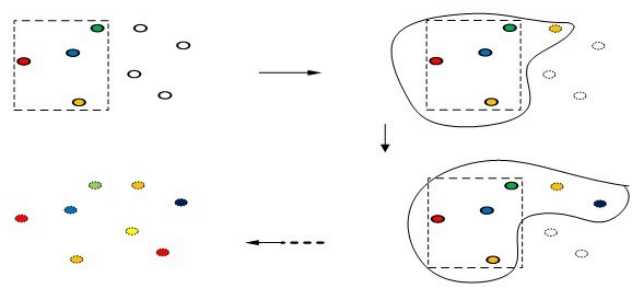

(d) Optimization with a part of the network remain unchanged (e.g. inaccessible)

Fig. 4: Various scenarios for channel assignment optimisation using the proposed approach

\section{EVALUATION AND RESULTS}

\section{A. Simulation Setup and Evaluation Strategy}

To evaluate the proposed channel assignment algorithm, we use MATLAB to simulate a dense Wi-Fi environment that consist of 50 APs randomly deployed in an area of $1200 \mathrm{~m} \times 1200 \mathrm{~m}$ at a minimum distance of 100 meters from each other, and the transmit power varying from $10 \mathrm{dBm}$ to $25 \mathrm{dBm}$. User stations are deployed randomly at a minimum distance of $1 \mathrm{~m}$ from each other. We adopted a large scale path loss model with the path loss exponent set to 2.5 , and a fixed noise level at $-95 \mathrm{dBm}$.

For our evaluation, we compare the performance of our SDN-based channel assignment algorithm against two common approaches, namely: Least Congested Channel (LCC) selection mechanism and uncoordinated channel assignment. In LCC, each AP acquires a suitable channel based on the neighbouring APs' channels [1] (this can also be implemented based on the contention and retransmission statistics evaluated at the MAC layer). In the uncoordinated approach, each AP randomly

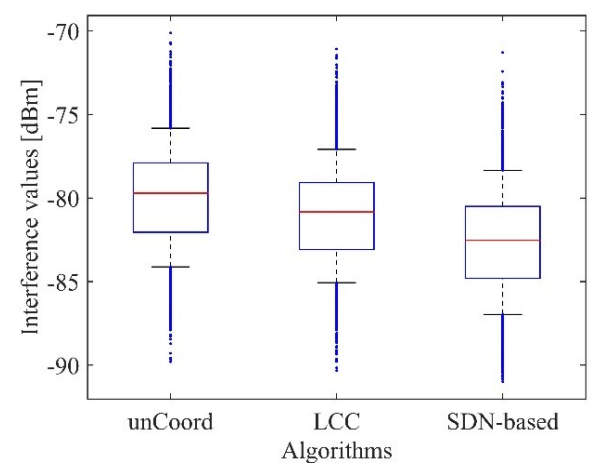

Fig. 5: Average interference levels obtained using each channel assignment algorithm acquires a channel without any prioritization, or with a prioritization over the orthogonal channels in some cases (e.g., channels 1, 6 or 11). This approach resembles a round robin assignment approach in dense Wi-Fi networks, where the number of APs is much larger than the number of available channels.

\section{B. Evaluation Results}

Fig. 5 shows the interference levels achieved throughout the network using each of the channel assignment algorithms considered in our evaluation. The upper and lower edges of the plotted boxes are the 25 th and 75 th percentile of the values and the median values are indicated by the central red lines. The values which we considered as outliers are indicated by blue dots in each case. The figure shows that our SDN-based approach results in better interference levels compared to the LCC and uncoordinated channel assignment approaches respectively. This include a consistent reduction of the interference of level for all of the monitored values, including the outliers, which results in a $3 \mathrm{~dB}$ reduction in the average interference level in the network compared to the uncoordinated approach and $2 \mathrm{~dB}$ reduction compared to the LCC approach.

Fig. 6 shows the Cumulative Distribution Function (CDF) of the SINR values measured at the users' stations using each of the channel assignment algorithms. This figure shows that our SDN-based algorithm outperforms uncoordinated approach and LCC by increasing the range of the achievable values of SINR and subsequently increasing the average SINR value (corresponding to $\mathrm{CDF}=0.5$ ) by $2.5 \mathrm{~dB}$ and $3.5 \mathrm{~dB}$ compared to the LCC and uncoordinated algorithms, respectively. The improvement in terms of SINR values seems slightly greater than the improvement depicted based on the interference values in Fig. 5. This is due to the impact of the improvement of SINR values over the SINR-based AP association process which is employed in our simulator. A lower interference value mostly influences the AP selection process for the users at the edge of 


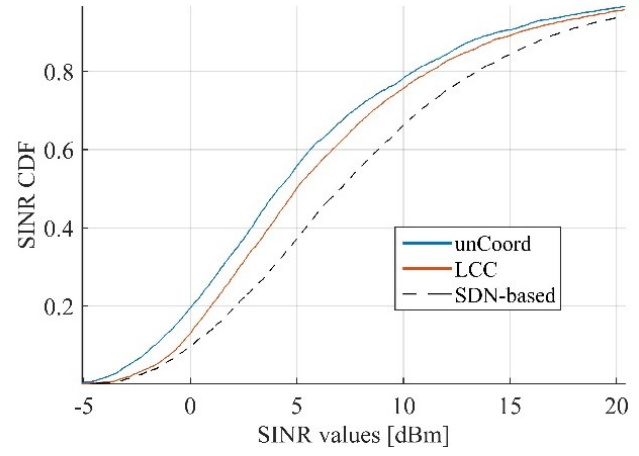

Fig. 6: CDF SINR obtained using each channel assignment algorithm

the APs' coverage area. By allowing some of these users to join an AP with a higher received signal strength (RSS), it intensifies the improvement of the performance through better AP selection.

The aim of the proposed SDN-based algorithm is to improve spectrum efficiency by reducing the interference through optimised channel assignment to the APs. This can be evaluated through the assessment of the spectrum utilization at the physical layer in terms of the achievable rate per unit of the employed bandwidth, b/s/Hz.

Fig. 7 shows the achieved spectral efficiency for the channel assignment algorithms considered in our evaluation. The upper and the lower sides of the thick parts of the depicted lines represent the range of the values from 1 st to the 3rd quartiles alongside the median value indicated at the middle of the lines. The outlier values are represented by the thin part of the lines. The obtained results presented in this figure show that our SDNbased algorithm outperforms LCC and uncoordinated algorithms by $0.4 \mathrm{~b} / \mathrm{s} / \mathrm{Hz}$ and $0.6 \mathrm{~b} / \mathrm{s} / \mathrm{Hz}$ on average, respectively. In terms of the channel capacity, these improvement are equal to $8 \mathrm{Mbps}$ and $12 \mathrm{Mbps}$ improvements in the achievable physical layer data rate compared to LCC and uncoordinated, respectively. This is based on a $20 \mathrm{MHz}$ channel bandwidth in IEEE802.11 2.4GHz. Furthermore, the proposed approach has expanded the range of the achievable spectral efficiency values to a higher level compared to LCC and uncoordinated algorithms.

\section{CONCLUSION}

In this paper we have presented a novel channel assignment algorithm that addresses spectrum congestion and interference in dense Wi-Fi environments. The proposed algorithm is based on an SDN-based Wi-Fi management framework where the controller acts as the central management entity upon which the assignment algorithm is executed. The algorithm relies on network status information such as the interference levels at each $\mathrm{AP}$, the current channel assignment configuration and the network topology. The performance of the algorithm has been assessed through simulation and the obtained results showed that it provides a lower interference, better SINR and a higher spectral efficiency within the network compared to the state of the art. Future work will consider introducing user-side quality information such as the received quality of the channel and QoS

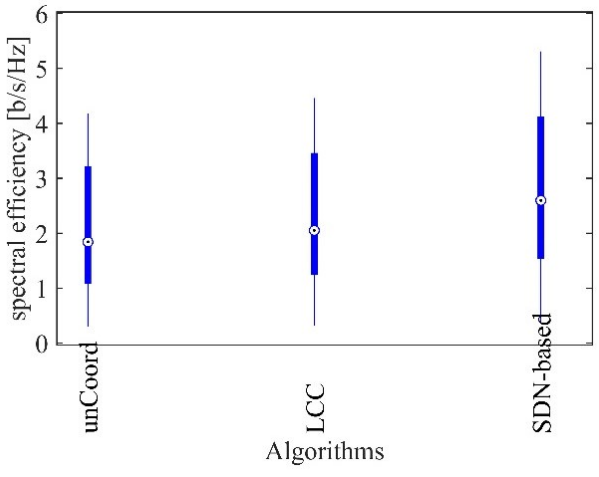

Fig. 7: Spectral efficiency using each channel assignment algorithm

metrics to further improve the performance of the channel assignment algorithm.

\section{ACKNOWLEDGEMENTS}

This work is funded by the Horizon 2020 Framework Programme of the European Union as part of the Wi-5 project (644262). Further information is available at http://www.wi5.eu/.

\section{REFERENCES}

[1] M. Achanta, "Method and Apparatus for Least Congested Channel Scan for Wireless Access Points," US Patent No. 20060072602, Apr. 2006

[2] K. Zhou, X. Jia, L. Xie, Y. Chang, and X. Tang, "Channel Assignment for WLAN by Considering Overlapping Channels in SINR Interference Model", International Conference on Computing, Networking and Communications (ICNC), Maui, Hawaii, USA 30 Jan.- 2 Feb. 2012.

[3] A. Mishra, S. Banerjee, and W. Arbaugh, "Weighted coloring based channel assignment for WLANs." SIGMOBILE Mob. Comput. Commun. Rev. 9(3): 19-31, 2005.

[4] Bondy, J.A., Murty, U.S.R., Graph Theory, Springer, ISBN 978-1-84628969-9, 2008.

[5] M. Yu, X. Ma, W. Su, and L. Tung, "A new joint strategy of radio channel allocation and power control for wireless mesh networks." Computer Communications 35(2): 196-206, 2012.

[6] Lingzhi Wang, Cunqing Hua, Rong Zheng and Rui Ni (2015). Online channel selection and user association in high-density WiFi networks. Communications (ICC), 2015 IEEE International Conference on.

[7] Riggio, R., M.K. Marina, and T. Rasheed, "Interference management in software-defined mobile networks," Integrated Network Management (IM), 2015 IFIP/IEEE International Symposium on.

[8] J. Schulz-Zander, et al., "OpenSDWN: programmatic control over home and enterprise WiFi", ACM SIGCOMM Symposium on Software Defined Networking Research, Santa Clara, CA, 14-17 Mar. 2016.

[9] R. Riggio, M. K. Marina, J. Schulz-Zander, S. Kuklinski and T. Rasheed, "Programming Abstractions for Software-Defined Wireless Networks," in IEEE Transactions on Network and Service Management, vol. 12, no. 2, pp. 146-162, June 2015

[10] J. Schulz-Zander, , et al., "Programmatic Orchestration of WiFi Networks," USENIX Symposium on Networked Systems Design and Implementation (NSDI), Philadelphia, PA, USA, 19-20 Jun. 2014.

[11] Wi-5 Project (What to do With the Wi-Fi Wild West), http://www.wi5.eu/ 